\title{
Robustness-based Design Optimization under Data Uncertainty
}

\author{
Kais Zaman, Mark McDonald, Sankaran Mahadevan* \\ Vanderbilt University, Nashville, TN, USA \\ and Lawrence Green \\ NASA Langley Research Center, Hampton, Virginia
}

\begin{abstract}
This paper proposes formulations and algorithms for design optimization under both aleatory (i.e., natural or physical variability) and epistemic uncertainty (i.e., imprecise probabilistic information), from the perspective of system robustness. The proposed formulations deal with epistemic uncertainty arising from both sparse and interval data without any assumption about the probability distributions of the random variables. A decoupled approach is proposed in this paper to un-nest the robustness-based design from the analysis of non-design epistemic variables to achieve computational efficiency. The proposed methods are illustrated for the upper stage design problem of a two-stage-toorbit (TSTO) vehicle, where the information on the random design inputs are only available as sparse point and/or interval data. As collecting more data reduces uncertainty but increases cost, the effect of sample size on the optimality and robustness of the solution is also studied. A method is developed to determine the optimal sample size for sparse point data that leads to the solutions of the design problem that are least sensitive to variations in the input random variables.
\end{abstract}

\section{Introduction}

In deterministic design optimization, it is generally assumed that all design variables and system variables are precisely known; the influence of natural variability and data uncertainty on the optimality and feasibility of the design is not explicitly considered. However, real-life engineering problems are non-deterministic, and a deterministic assumption about inputs may lead to infeasibility or poor performance (Sim, 2006). In recent years, many methods have been developed for design under uncertainty. Reliability-based design (e.g., Chiralaksanakul and Mahadevan, 2005; Ramu et al, 2006; Agarwal et al, 2007and Du and Huang, 2007) and robust design (e.g., Parkinson et al,

* Corresponding author: sankaran.mahadevan@vanderbilt.edu 
1993; Du and Chen, 2000; Doltsinis and Kang, 2004 and Huang and Du, 2007) are two directions pursued by these methods. While reliability-based design aims to maintain design feasibility at desired reliability levels, robust design optimization attempts to minimize variability in the system performance due to variations in the inputs (Lee et al, 2008). In recent years, several methods have also been proposed to integrate these two paradigms of design under uncertainty (e.g., Du et al, 2004, Lee et al, 2008).

Taguchi proposed robust design methods for selecting design variables in a manner that makes the product performance insensitive to variations in the manufacturing process (Taguchi, 1993). Taguchi's methods have widespread applications in engineering; however, these methods are implemented through statistical design of experiments and cannot solve problems with multiple measures of performances and design constraints (Wei et al, 2009). With the introduction of nonlinear programming to robust design, it has become possible to achieve robustness in both performance and design constraints ( $\mathrm{Du}$ and Chen, 2000).

The essential elements of robust design optimization are: (1) maintaining robustness in the objective function (objective robustness); (2) maintaining robustness in the constraints (feasibility robustness); (3) estimating mean and measure of variation (variance) of the performance function; and (4) multi-objective optimization. The rest of this section briefly reviews the literature with respect to these four elements and establishes the motivation for the current study.

Objective robustness

In robust optimization, the robustness of the objective function is usually achieved by simultaneously optimizing its mean and minimizing its variance. Two major robustness 
measures are available in the literature: one is the variance, which is extensively discussed in the literature (Du and Chen, 2000; Lee and Park, 2001 and Doltsinis and Kang, 2004) and the other is based on the percentile difference (Du et al, 2004). Although the percentile difference method has the advantage that it contains the information of probability in the tail regions of the performance distribution, this method is only applicable to unimodal distributions. Variance as a measure of variation of the performance function can be applied to any distribution (unimodal or multimodal), but it only characterizes the dispersion around the mean (Huang and $\mathrm{Du}, 2007$ ).

\section{$\underline{\text { Feasibility robustness }}$}

Feasibility robustness i.e., robustness in the constraints can be defined as satisfying the constraints of the design in the presence of uncertainty. Du and Chen (2000) classified the methods of maintaining feasibility robustness into two categories, methods that use probabilistic and statistical analysis, and methods that do not require them. Among the methods that require probabilistic and statistical analysis, a probabilistic feasibility formulation (Du and Chen, 2000 and Lee et al, 2008), and a moment matching formulation (Parkinson et al, 1993) have been proposed. Du and Chen (2000) used a most probable point (MPP)-based importance sampling method to reduce the computational burden associated with the probabilistic feasibility formulation. The moment matching formulation is a simplified approach which requires only the constraints on the first and second moments of the performance function to be satisfied, and assumes that the performance function is normally distributed. A variation of this approach, the feasible region reduction method has been described in Park et al (2006), which is more general and does not require the normality assumption. This is a tolerance design method, where 
width of the feasible space in each direction is reduced by the amount $k \sigma$, where $k$ is a user-defined constant and $\sigma$ is the standard deviation of the performance function. This method only requires the mean and variance of the performance function.

Methods that do not require probabilistic and statistical analysis are also available, for example, worst case analysis (Parkinson et al, 1993), corner space evaluation (Sundaresan et al, 1995), and manufacturing variation patterns (MVP) (Yu and Ishii, 1998). A comparison study of the different constraint feasibility methods can be found in Du and Chen (2000).

Estimating mean and variance of the performance function

Various methods have been reported in the literature to estimate the mean and standard deviation of the performance function. These methods can be divided into three major classes: (i) Taylor series expansion methods, (ii) sampling-based methods and (iii) point estimate methods (Huang and $\mathrm{Du}, 2007$ ).

The Taylor series expansion method (Haldar and Mahadevan, 2000; Du and Chen, 2000; and Lee et al, 2001) is a simple approach. However, for a nonlinear performance function, if the variances of the random variables are large, this approximation may result in large errors (Du et al., 2004). Although a second-order Taylor series expansion is generally more accurate than the first-order approximation, it is also computationally more expensive.

Sampling-based methods require information on distributions of the random variables, and are expensive. Efficient sampling techniques such as importance sampling, Latin hypercube sampling, etc. (Robert and Cesalla, 2004) can be used to reduce the computational effort, but are still prohibitive in the context of optimization. Surrogate 
models (Ghanem and Spanos 1991; Bichon et al, 2008; Cheng and Sandu, 2009) may be used to further reduce computational effort.

In an attempt to overcome the difficulties associated with the computation of derivatives required in Taylor series expansion, Rosenlblueth (1975) proposed a point estimate method to compute the first few moments of the performance function. Different variations of this point estimate method (Hong, 1998; Zhao and Ono, 2000 and Zhao and Ang, 2003) have been studied. Although point estimate methods are easier to implement, the accuracy may be low and may generate points that lie outside the domain of the random variable.

\section{Multi-objective optimization}

Robustness-based optimization considers two objectives: optimize the mean of the objective function and minimize its variation. An extensive survey of the multi-objective optimization methods can be found in Marler and Arora (2004). Among the available methods, the weighted sum approach is the most common approach to multi-objective optimization and has been extensively used in robust design optimization (Lee and Park, 2001; Doltsinis and Kang, 2004; Zou and Mahadevan, 2006). The designer can obtain alternative design points by varying the weights and can select the one that offers the best trade-off among multiple objectives. Despite its simplicity, the weighted sum method may not obtain potentially desirable solutions (Park et al, 2006). Another common approach is the $\varepsilon$-constraint method in which one of the objective functions is optimized while the other objective functions are used as constraints. Despite its advantages over weighted sum method in some cases, the $\varepsilon$-constraint method can be computationally expensive for more than two objective functions (Mavrotas, 2009). 
Other methods include goal programming (Zou and Mahadevan, 2006), compromise decision support problem (Bras and Mistree, 1993, 1995; Chen et al, 1996), compromise programming (CP) (Zalney, 1973; Zhang, 2003; Chen et al, 1999) and physical programming (Messac, 1996; Messac et al, 2001; Messac and Ismail-Yahaya, 2002; Chen et al, 2000). Each of these methods has its own advantages and limitations.

Although there is now an extensive volume of literature for robust optimization methods and applications, all these methods have only been studied with respect to physical or natural variability represented by probability distributions. Uncertainty in system design also arises from other contributing factors. Sources of uncertainty may be divided into two types: aleatory and epistemic (Oberkampf et. al., 2004). Aleatory uncertainty is irreducible. Examples include phenomena that exhibit natural variation like operating conditions, material properties, geometric tolerances, etc. In contrast, epistemic uncertainty results from a lack of knowledge about the system, or due to approximations in the system behavior models, or due to limited or subjective (e.g., expert opinion) data; it can be reduced as more information about the system is obtained.

One type of data uncertainty involves having limited data to properly define the distribution parameters of the random variables. This type of uncertainty may be reduced by collecting more data. In some cases of data uncertainty, distribution information of a random variable may only be available as intervals given by experts. The objective of this paper is to develop an efficient robust optimization methodology that includes both aleatory and epistemic uncertainty described through sparse point data and interval data.

A few studies on robust design optimization are reported in the literature to deal with epistemic uncertainty arising from lack of information. Youn et al (2007) used a 
possibility-based method, and redefined the performance measure of robust design using the most likely values of fuzzy random variables. Dai and Mourelatos (2003) proposed two two-step methods for robust design optimization that can treat aleatory and epistemic uncertainty separately using a range method and a fuzzy sets approach. Most of the current methods of robust optimization for epistemic uncertainty need additional nonprobabilistic formulations to incorporate epistemic uncertainty into the robust optimization framework, which may be computationally expensive. However, if the epistemic uncertainty can be converted to a probabilistic format, the need for these additional formulations is avoidable, and well-established probabilistic methods of robust design optimization can be used. Therefore, there is a need for an efficient robust design optimization methodology that deals with both aleatory and epistemic uncertainty.

In this paper, we propose robustness-based design optimization formulations that work under both aleatory and epistemic uncertainty using probabilistic representations of different types of uncertainty. Our proposed formulations deal with both sparse point and interval data without any assumption about probability distributions of the random variables.

The performance of robustness-based design can be defined by the mean and variation of the performance function. In our proposed formulations, we obtain the optimum mean value of the objective function (e.g., gross weight) while also minimizing its variation (e.g., standard deviation). Thus, the design will meet target values in terms of both design bounds and standard deviations of design objectives and design variables thereby ensure feasibility robustness. 
A Taylor series expansion method is used in this paper to estimate the mean and standard deviation of the performance function, which requires means and standard deviations of the random variables. However, with sparse point data and interval data, it is impossible to know the true moments of the data, and there are many possible probability distributions that can represent these data (Zaman et al, 2009). In this paper, we propose methods for robustness-based design optimization that account for this uncertainty in the moments due to sparse point data and interval data and thereby include epistemic uncertainty into the robust design optimization framework. As collecting more data reduces uncertainty but increases cost, the effect of sample size on the optimality and the robustness of the solution is also studied. A method to determine the optimal sample size for sparse point data that will lead to the minimum scatter on solutions to the design problem is also presented in this paper.

In some existing methods for robust design under epistemic uncertainty, all the epistemic variables are considered as design variables (Youn et al, 2007). However, if the designer does not have any control on an epistemic variable (e.g., Young's modulus in beam design), considering that variable as a design variable might lead to a solution that could underestimate the design objectives. Therefore, in this paper, we propose a general formulation for robust design that considers some of the epistemic variables as nondesign variables, which leads to a conservative design under epistemic uncertainty.

Note that the proposed robustness-based design optimization method is general and capable of handling a wide range of application problems under data uncertainty. The proposed methods are illustrated for the conceptual level design process of a two-stage- 
to-orbit (TSTO) vehicle, where the distributions of the random inputs are described by sparse point and/or interval data.

The rest of the paper is organized as follows. Section 2 proposes robustness-based design optimization framework for sparse point data and interval data. In Section 3, we illustrate the proposed methods for the conceptual level design process of a TSTO vehicle. Section 4 provides conclusions and suggestions for future work.

\section{Proposed methodology}

\section{Deterministic design optimization}

In a deterministic optimization formulation, all design variables and system variables are considered deterministic. No random variability or data uncertainty is taken into account. The deterministic optimization problem is formulated as follows:

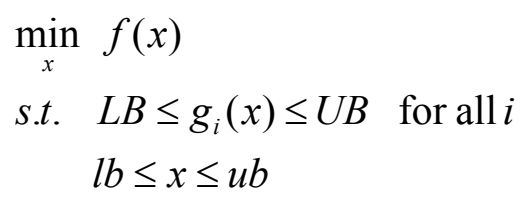

where $f(x)$ is the objective function, $x$ is the vector of design variables, $g_{i}(x)$ is the $i$ th constraint, $L B$ and $U B$ are the vectors of lower and upper bounds of constraints $g_{i}{ }^{\prime} s$ and $l b$ and $u b$ are the vectors of lower and upper bounds of design variables.

In practice, the input variables might be uncertain and solutions of this deterministic formulation could be sensitive to the variations in the input variables. Robustness-based design optimization takes this uncertainty into account. The optimal design points obtained using the deterministic method could be used as initial guesses in robustness-based optimization. 


\section{$\underline{\text { Robustness-based design optimization }}$}

In the proposed methodology, we use variance as a measure of variation of the performance function in order to achieve objective robustness, the feasible region reduction method to achieve feasibility robustness, a first-order Taylor series expansion to estimate the mean and variance of the performance function, and a weighted sum method for the aggregation of multiple objectives. This combination of methods is only used for the sake of illustration. Other approaches can be easily substituted in the proposed methodology. The robustness-based design optimization problem can now be formulated as follows:

$$
\begin{array}{ll}
\min _{d} & f(\mu, \sigma)=\left(w^{*} \mu_{f}+v^{*} \sigma_{f}\right) \\
\text { s.t. } & L B+k \sigma\left(g_{i}(d, z)\right) \leq E\left(g_{i}(d, z)\right) \leq U B-k \sigma\left(g_{i}(d, z)\right) \text { for all } i \\
& l b+k \sigma\left(x_{i}\right) \leq d_{i} \leq u b-k \sigma\left(x_{i}\right) \quad \text { for } i=1,2, \ldots, n r d v \\
& l b \leq d_{i} \leq u b \quad \text { for } i=1,2, \ldots, n d d v
\end{array}
$$

where $\boldsymbol{d}$ is the vector of deterministic design variables as well as the mean values of the uncertain design variables $\boldsymbol{x} ; n r d v$ and $n d d v$ are the numbers of the random design variables and deterministic design variables, respectively; and $\mathbf{z}$ is the vector of nondesign input random variables, whose values are kept fixed at their mean values as a part of the design. The weighting coefficients $w \geq 0$ and $v \geq 0$ represent the relative importance of the objectives $\mu_{f}$ and $\sigma_{f}$ in Eq. (2); $g_{i}(d, z)$ is the $i$ th constraint; $E\left(g_{i}(d, z)\right)$ is the mean and $\sigma\left(g_{i}(d, z)\right)$ is the standard deviation of the $i$ th constraint. $L B$ and $U B$ are the vectors of lower and upper bounds of constraints $g_{i}{ }^{\prime} s ; l b$ and $u b$ are the vectors of lower and upper bounds of the design variables; $\sigma(x)$ is the vector of standard 
deviations of the random variables and $k$ is some constant. The role of the constant $k$ is to adjust the robustness of the method against the level of conservatism of the solution. It reduces the feasible region by accounting for the variations in the design variables and is related to the probability of constraint satisfaction. For example, if a design variable or a constraint function is normally distributed, $k=1$ corresponds to the probability $0.8413, k$ $=2$ to the probability 0.9772 , etc.

Note that the robust design formulation in Eq. (2) is a standard nonlinear multiobjective optimization formulation. The optimality conditions of such a formulation have been extensively described in the literature including Cagan and Williams (1993) and Marler and Arora (2004).

In the proposed formulation, the performance functions considered are in terms of the model outputs. The means and standard deviations of the objective and constraints are estimated by using a first-order Taylor series approximation as follows:

Performance function: $Y=g\left(X_{1}, X_{2}, \ldots, X_{n}\right)$

First-order approximate mean of y: $E\left(Y^{\prime}\right) \approx g\left(\mu_{X_{1}}, \mu_{X_{2}}, \ldots ., \mu_{X_{n}}\right)$

First-order variance of y: $\operatorname{Var}\left(Y^{\prime}\right) \approx \sum_{i=1}^{n}\left(\frac{\partial g}{\partial X_{i}}\right)^{2} \operatorname{Var}\left(X_{i}\right)+\sum_{i=1}^{n} \sum_{j=1}^{n} \frac{\partial g}{\partial X_{i}} \frac{\partial g}{\partial X_{j}} \operatorname{Cov}\left(X_{i}, X_{j}\right)$

The implementation of Eq. (2) requires that variances of the random design variables $X_{i}$ and the means and variances of the random non-design variables $Z_{i}$ be precisely known, which is possible only when a large number of data points are available. In practical situations, only a small number of data points may be available for the input variables. In other cases, information about random input variables may only be specified as intervals, as by expert opinion. This is input data uncertainty, causing uncertainty 
regarding the distribution parameters (e.g., mean and variance) of the inputs $X_{i}$ and $Z_{i}$. Robustness-based optimization has to take this into account. In the following subsections, we propose a new methodology for robustness-based design optimization that accounts for data uncertainty.

\subsection{Robustness-based design optimization under data uncertainty}

The inclusion of epistemic uncertainty in robust design adds another level of complexity in the design methodology. The design variables $\boldsymbol{d}$ and/or the input random variables $\mathbf{z}$ in Eq. (2) might have epistemic uncertainty. Since the designer does not have any control on the non-design epistemic variables $\mathbf{z}$, the design methodology has to employ a search among the possible values of such epistemic variables in order to find an optimal solution. In such case, we get a conservative robust design. The robustness-based design optimization problem can now be formulated with the following generalized statement:

$$
\begin{array}{ll}
\max _{\mu_{z}}\left(\min _{d}\right. & \left.f(\mu, \sigma)=\left(w^{*} \mu_{f}+v^{*} \sigma_{f}\right)\right) \\
\text { s.t. } & L B+k \sigma\left(g_{i}\left(d, \mu_{z}\right)\right) \leq E\left(g_{i}(d, z)\right) \leq U B-k \sigma\left(g_{i}\left(d, \mu_{z}\right)\right) \text { for all } i \\
& l b+k \sigma(x) \leq d \leq u b-k \sigma(x) \\
& Z_{l} \leq \mu_{z} \leq Z_{u}
\end{array}
$$

where $Z_{l}$ and $Z_{u}$ are the vectors of lower and upper bounds of the decision variables $\mu_{z}$ of the outer loop optimization problem.

Note that in this formulation, the inner loop decision variables $\boldsymbol{d}$ may consist of stochastic design variables as well as epistemic design variables. The inner loop optimization is a design optimization problem, where a robust design optimization is carried out for a fixed 
set of non-design epistemic variables. The outer loop optimization is the analysis for the non-design epistemic variables, where the optimizer searches among the possible values of the non-design epistemic variables for a conservative solution of the robust design problem.

This nested optimization problem can be decoupled and expressed as:

$$
\begin{aligned}
& d^{*}=\underset{d}{\arg \min }\left(w^{*} \mu_{f}\left(d, \mu_{z}^{*}\right)+v^{*} \sigma_{f}\left(d, \mu_{z}^{*}\right)\right) \\
& \text { s.t. } \quad L B+k \sigma\left(g_{i}\left(d, \mu_{z}^{*}\right)\right) \leq E\left(g_{i}(d, z)\right) \leq U B-k \sigma\left(g_{i}\left(d, \mu_{z}^{*}\right)\right) \text { for all } i \\
& l b+k \sigma(x) \leq d \leq u b-k \sigma(x) \\
& \mu_{z}^{*}=\underset{\mu_{z}}{\arg \max }\left(w^{*} \mu_{f}\left(d^{*}, \mu_{z}\right)+v^{*} \sigma_{f}\left(d^{*}, \mu_{z}\right)\right) \\
& \text { s.t. } \quad L B+k \sigma\left(g_{i}\left(d^{*}, \mu_{z}\right)\right) \leq E\left(g_{i}(d, z)\right) \leq U B-k \sigma\left(g_{i}\left(d^{*}, \mu_{z}\right)\right) \text { for all } i \\
& Z_{l} \leq \mu_{z} \leq Z_{u}
\end{aligned}
$$

The optimization problems in Eqs. (7) and (8) are solved iteratively until convergence. Note that the first constraint (i.e., the robustness constraint) in Eq. (8) is required to ensure that the optimization is driven by all non-design epistemic variables, because sometimes the objective function may not be a function of all non-design epistemic variables. In cases when the objective function is the function of all non-design epistemic variables, this constraint is not required.

\subsubsection{Robustness-based design with sparse point data}

In this section, we propose a methodology for robustness-based design optimization with sparse point data. It is assumed that only sparse point data are available for the uncertain design variables as well as non-design epistemic variables. 
Since the data size is small, there is uncertainty about the mean and variance calculated from the samples. The chi-square distribution is a good assumption for the distribution of the variance, if the underlying population is normal. The two-sided (1- $\alpha)$ confidence interval for the population variance $\sigma^{2}$ can be expressed as (Haldar and Mahadevan, 2000):

$\left[\frac{(n-1) s^{2}}{c_{1-\alpha / 2, n-1}} ; \frac{(n-1) s^{2}}{c_{\alpha / 2, n-1}}\right]$

where $n$ is the sample size, $s$ is the sample standard deviation of sparse point data, and $c_{\alpha / 2, n-1}$ is obtained from the chi-square distribution at $(n-1)$ degrees of freedom and $\alpha$ significance level. Note that Eq. (9) can still be used to obtain approximate confidence bounds for variance if the underlying population is not normal. However, in such cases, other approximation methods (Bonett, 2006; Cojbasic and Tomovic, 2007) can be used to obtain more reliable estimates of confidence bounds. In robustness-based design optimization, we are interested in obtaining a solution that is least sensitive to the variations in the input random variables; therefore we use the upper bound variances for the input random variables $x_{i}$ and $z_{i}$ to solve the formulations in Eqs. (7)-(8) for sparse point data.

For non-design epistemic variables described by sparse point data, the constraints on the decision variables in Eq. (8) are implemented through the construction of confidence intervals about means. As the design variables are described by the sparse point data, it is possible that the underlying distributions of the design variables might have major deviations from normality. Therefore, we have used the Johnson's modified $t$ 
statistic (Johnson, 1978) to construct the confidence bounds on means of the design variables as follows:

$$
\begin{aligned}
& Z_{l}=\bar{z}-t_{\alpha / 2, n-1} \frac{s}{\sqrt{n}}+\frac{\mu_{3}}{6 s^{2} n} \\
& Z_{u}=\bar{z}+t_{\alpha / 2, n-1} \frac{s}{\sqrt{n}}+\frac{\mu_{3}}{6 s^{2} n}
\end{aligned}
$$

where $\bar{z}$ is the vector of means of the epistemic variables, $s$ is the vector of standard deviations, $n$ is the sample size of the sparse point data, $\mu_{3}$ is the third central moment and $t_{\alpha / 2, n-1}$ is obtained from the Student $t$ distribution at $(n-1)$ degrees of freedom and $\alpha$ significance level. This modified statistic takes into account the skewness of the distribution and thus provides a better estimate of the confidence bound in the presence of limited data.

The optimization formulation shown in Eqs. (7)-(8) involves aggregation of multiple objectives. In the proposed formulations, the aggregate objective function consists of two types of objectives, expectation and standard deviation of model outputs. Since different objectives have different magnitudes, a scaling factor has to be used in the formulation.

\subsubsection{Determination of optimal sample size for sparse point data}

The optimal solutions depend on the sample size of the sparse data as will be discussed in Section 3.1. Therefore, it is of interest to determine the optimal sample size of the sparse data that leads to the solution of the design problem that is least sensitive to the variations of design variables. This will facilitate resource allocation decisions for data collection. The following two optimization formulations are solved iteratively until 
convergence for the optimal sample sizes of the epistemic design variables $\left(n_{d}^{*}\right)$ and epistemic non-design variables $\left(n_{e}^{*}\right)$.The formulations in Eq. (11)-(12) are the weighted sum formulations of a three-objective optimization problem, where the first and second objectives are the mean and standard deviation of GW respectively and the third objective is the total cost of obtaining samples for all the random variables.

$$
\begin{aligned}
& {\left[d^{*}, n_{d}^{*}\right]=\arg \min _{d, n_{d}}\left(w^{*} E\left(g_{i}\left(d, \mu_{z}^{*}\right)\right)+v^{*} \sigma\left(g_{i}\left(d, \mu_{z}^{*}\right), n_{d}, n_{e}^{*}\right)+(1-w-v) *\left(\sum_{j=1}^{m} n_{d_{j}} c_{d j}+\sum_{j=1}^{q} n_{e_{j}}^{*} c_{e_{j}}\right)\right)} \\
& \text { s.t. } \quad L B+k \sigma\left(g_{i}\left(d, \mu_{z}^{*}\right), n_{d}, n_{e}^{*}\right) \leq E\left(g_{i}(d, z)\right) \leq U B-k \sigma\left(g_{i}\left(d, \mu_{z}^{*}\right), n_{d}, n_{e}^{*}\right) \text { for all } i \\
& l b+k \sigma\left(x, n_{d}\right) \leq d \leq u b-k \sigma\left(x, n_{d}\right) \\
& \sum_{j=1}^{m} n_{d_{j}} c_{d_{j}}+\sum_{j=1}^{q} n_{e_{j}}^{*} c_{e_{j}} \leq C \\
& n_{d j} \leq b_{d j} \quad \text { for all } \mathrm{j} \\
& {\left[\mu_{z}^{*}, n_{e}^{*}\right]=\arg \max _{\mu_{z}, n_{e}}\left(w^{*} E\left(g_{i}\left(d^{*}, \mu_{z}\right)\right)+v^{*} \sigma\left(g_{i}\left(d^{*}, \mu_{z}\right), n_{d}^{*}, n_{e}\right)+(1-w-v) *\left(\sum_{j=1}^{m} n_{d_{j}}^{*} c_{d j}+\sum_{j=1}^{q} n_{e_{j}} c_{e_{j}}\right)\right)} \\
& \text { s.t. } \quad L B+k \sigma\left(g_{i}\left(d^{*}, \mu_{z}\right), n_{d}^{*}, n_{e}\right) \leq E\left(g_{i}(d, z)\right) \leq U B-k \sigma\left(g_{i}\left(d^{*}, \mu_{z}\right), n_{d}^{*}, n_{e}\right) \text { for all } i \\
& Z_{l}\left(n_{e}\right) \leq \mu_{z} \leq Z_{u}\left(n_{e}\right) \\
& \sum_{j=1}^{m} n_{d_{j}}^{*} c_{d_{j}}+\sum_{j=1}^{q} n_{e_{j}} c_{e_{j}} \leq C \\
& n_{e_{j}} \leq b_{e \mathrm{j}} \quad \text { for all } \mathrm{j}
\end{aligned}
$$

where $w \geq 0$ and $v \geq 0$ are the weighting coefficients that represent the relative importance of the objectives; $n_{d_{j}}$ and $n_{e_{j}}$ are the sample sizes and $b_{d_{j}}$ and $b_{e_{j}}$ are the maximum sample size possible for the $j$ th design and non-design random variables, respectively. The number of design and non-design random variables are denoted by $m$ and $q$, respectively. Terms $c_{d_{j}}$ and $c_{e_{j}}$ are the cost of obtaining one sample for the $j$ th 
random design and non-design variables, respectively and $C$ is the total cost allocated for obtaining samples for all the random variables. Note that as in Eq. (8), the robustness constraint in Eq. (12) is only required if the objective function is not a function of all nondesign epistemic variables. The optimization formulation presented above is a mixedinteger nonlinear problem. A relaxed problem is solved in Section 3.

\subsubsection{Robustness-based design with interval data}

This section proposes a methodology for robustness-based design optimization with interval data. In this case, the only information available for one or more input random variables is in the form of single interval or multiple interval data. The following discussion develops a methodology to solve the formulations in Eq. (7)-(8) for uncertainty represented through interval data.

For interval data, the moments (e.g., mean and variance) are not a single value, rather only bounds can be given (Zaman et al, 2009). We have proposed methods to compute the bounds of moments for both single and multiple interval data in Zaman et al (2009). The methods for computing bounds of the first two moments for interval data are given later in this section. Once the bounds on the mean and variance of interval data are estimated, we use the upper bounds of sample variance to solve the formulations of robust design under uncertainty represented through single interval or multiple interval data. Therefore, the resulting solution becomes least sensitive to the variations in the uncertain variables. 
For non-design epistemic variables described by interval data, the constraints on the decision variables in Eqs. (8) and (12) are implemented through estimating the bounds of the means by the methods as described later in this section.

The following discussions briefly summarize the methods to estimate the bounds on the first two moments for single interval and multiple interval data, respectively.

\section{Bounds on moments with single interval data}

The methods for calculating bounds on the first two moments for single interval data are summarized in Table 1 below.

Table 1: Methods for calculating moment bounds for single interval data

\begin{tabular}{|c|c|c|c|}
\hline \multirow{2}{*}{ Moment } & Lower bound & Fpper bound & Formula \\
\cline { 2 - 3 } 1 & $\begin{array}{r}\mathrm{PMF}=1 \text { at lower endpoint } \\
=0 \text { elsewhere }\end{array}$ & $\begin{array}{c}\mathrm{PMF}=1 \text { at upper endpoint } \\
=0 \text { elsewhere }\end{array}$ & $M_{1}=E(x)$ \\
\hline 2 & $\begin{array}{r}\mathrm{PMF}=1 \text { at any point } \\
=0 \text { elsewhere }\end{array}$ & $\begin{array}{c}\mathrm{PMF}=0.5 \text { at each } \\
\text { endpoint }\end{array}$ & $M_{2}=E\left(x^{2}\right)-(E(x))^{2}$ \\
\hline
\end{tabular}

In Table 1, the formulas lead to the lower and upper endpoints of the interval as the lower and upper bounds for the first moment, respectively. The formulas also imply that the lower bound for the second moments is zero.

\section{Bounds on moments with multiple interval data}

The methods for calculating bounds on the first two moments for multiple interval data are summarized in Table 2 below. 
Table 2: Methods for calculating moment bounds for multiple interval data

\begin{tabular}{|c|c|}
\hline Moment & Formula \\
\hline 1 & {$\left[\begin{array}{ll}\underline{M} & \bar{M}\end{array}\right]=\left[\frac{1}{n} \sum_{i}^{n} l b_{i}, \frac{1}{n} \sum_{i}^{n} u b_{i}\right]$} \\
\hline 2 & $\begin{array}{ll}\min _{x_{1}, \ldots, x_{n}} / \max & M_{2}=\frac{1}{n} \sum_{i=1}^{n}\left(x_{i}-\frac{1}{n} \sum_{j=1}^{n} x_{j}\right) \\
\text { s.t. } & l b_{i} \leq x_{i} \leq u b_{i} \quad i=\{1, \ldots, n\}\end{array}$ \\
\hline
\end{tabular}

Note: $\left[\begin{array}{ll}l b_{i} & u b_{i}\end{array}\right]=$ Set of intervals $n=$ Number of intervals

Once the bounds on the mean and variance of interval data are estimated by the methods described above, we can now use these bounds to solve the formulations of robustness-based design optimization under uncertainty represented through single interval or multiple interval data. In the following section, we illustrate our proposed formulations for robustness-based design optimization with both sparse point and interval data.

\section{Example Problem}

In this section, the proposed methods are illustrated for the conceptual level design process of a TSTO vehicle. The multidisciplinary system analysis consists of geometric modeling, aerodynamics, aerothermodynamics, engine performance analysis, trajectory analysis, mass property analysis and cost modeling (Stevenson et al, 2002). In this paper, a simplified version of the upper stage design process of a TSTO vehicle is used to illustrate the proposed methods. High fidelity codes of individual disciplinary analysis are replaced by inexpensive surrogate models. Figure 1 illustrates the analysis process of a TSTO vehicle. 


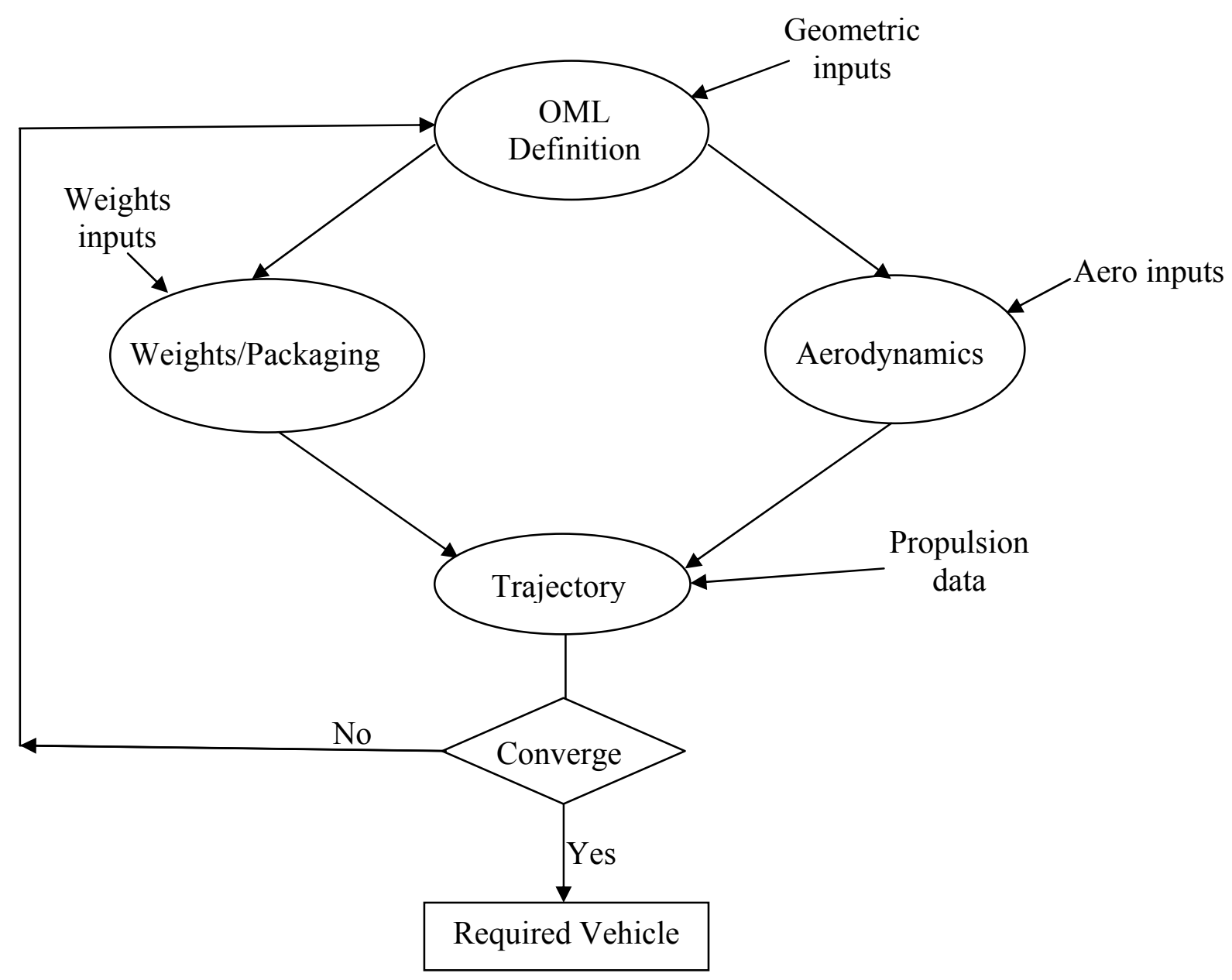

Figure 1: TSTO vehicle concept

The analysis outputs (performance functions) are Gross Weight (GW), Engine Weight (EW), Propellant Fraction Required (PFR), Vehicle Length (VL), Vehicle Volume (VV) and Body Wetted Area (BWA). Each of the analysis outputs is approximated by a second-order response surface and is a function of the random design variables Nozzle Expansion Ratio (ExpRatio), Payload Weight (Payload), Separation Mach (SepMach), Separation Dynamic Pressure (SepQ), Separation Flight Path Angle (SepAngle), and 
Body Fineness Ratio (Fineness). Each of the random variables is described by either sparse point data or interval data.

The objective is to optimize an individual analysis output (e.g., Gross Weight) while satisfying the constraints imposed by each of the design variables as well as all the analysis outputs. We note here that we have assumed independence among the uncertain input variables and thereby ignored the covariance terms in Eq. (5) to estimate the variance of the performance function in each of the following examples. The numerical values of the design bounds for the design variables and analysis outputs are given in Tables 3 and 4, respectively.

Table 3: Design bounds for the design variables

\begin{tabular}{|c|c|c|}
\hline Design Variable & $\mathrm{lb}$ & $\mathrm{ub}$ \\
\hline ExpRatio & 40 & 150 \\
\hline Payload & 8000 & 40000 \\
\hline SepMach & 7 & 12 \\
\hline SepQ & 40 & 200 \\
\hline SepAngle & 7 & 12 \\
\hline Fineness & 4 & 6 \\
\hline
\end{tabular}

Table 4: Design bounds for the analysis outputs

\begin{tabular}{|c|c|c|}
\hline Analysis output & LB & UB \\
\hline GW & 0 & $100 \mathrm{e}+005$ \\
\hline EW & 0 & $100 \mathrm{e}+005$ \\
\hline PFR & 0.4 & 0.95 \\
\hline VL & 0 & $100 \mathrm{e}+002$ \\
\hline VV & 0 & $100 \mathrm{e}+003$ \\
\hline BWA & 0 & $100 \mathrm{e}+005$ \\
\hline
\end{tabular}

\subsection{Robustness-based design optimization with sparse point data}

The methodology proposed in Section 2.1.1 is illustrated here for the TSTO problem. It is assumed that all the input variables $\boldsymbol{x}$ are described by sparse point data as 
given in Table 5. For this example, the input variable SepQ is assumed to be a non-design epistemic variable and all the remaining variables are assumed to be design variables. The design bounds for the respective design variables and the analysis outputs are given in Tables 3 and 4.

Table 5: Sparse Point Data for the random design variables

\begin{tabular}{|c|c|c|c|c|c|c|}
\hline Sample & ExpRatio & Payload & SepMach & SepQ & SepAngle & Fineness \\
\hline 01 & 85.23 & $2.8952 \mathrm{e}+004$ & 10.85 & 115.38 & 9.12 & 4.07 \\
\hline 02 & 82.25 & $2.9747 \mathrm{e}+004$ & 10.56 & 11.63 & 9.49 & 4.02 \\
\hline 03 & 88.79 & $2.6638 \mathrm{e}+004$ & 10.93 & 118.57 & 9.85 & 4.47 \\
\hline 04 & 83.93 & $2.8356 \mathrm{e}+004$ & 10.70 & 111.60 & 9.87 & 4.15 \\
\hline 05 & 80.67 & $2.7193 \mathrm{e}+004$ & 10.58 & 100.34 & 9.27 & 4.15 \\
\hline 06 & 91.32 & $2.9168 \mathrm{e}+004$ & 10.82 & 102.42 & 9.21 & 4.17 \\
\hline 07 & 83.64 & $2.8844 \mathrm{e}+004$ & 10.88 & 117.25 & 9.57 & 4.23 \\
\hline 08 & 86.64 & $2.5836 \mathrm{e}+004$ & 10.99 & 109.69 & 9.64 & 4.32 \\
\hline 09 & 90.32 & $2.9310 \mathrm{e}+004$ & 10.00 & 116.90 & 9.42 & 4.01 \\
\hline 10 & 85.39 & $2.9949 \mathrm{e}+004$ & 10.87 & 104.19 & 9.21 & 4.42 \\
\hline
\end{tabular}

The design problem becomes:

$$
\begin{array}{ll}
d^{*}=\underset{d}{\arg \min }\left(w^{*} E(G W)+(1-w) * \sigma(G W)\right) \\
\text { s.t. } \quad L B_{1}+k \sigma(G W) \leq E(G W) \leq U B_{1}-k \sigma(G W) \\
& L B_{2}+k \sigma(E W) \leq E(E W) \leq U B_{2}-k \sigma(E W) \\
& L B_{3}+k \sigma(P F R) \leq E(P F R) \leq U B_{3}-k \sigma(P F R) \\
& L B_{4}+k \sigma(V L) \leq E(V L) \leq U B_{4}-k \sigma(V L) \\
& L B_{5}+k \sigma(V V) \leq E(V V) \leq U B_{5}-k \sigma(V V) \\
& L B_{6}+k \sigma(B W A) \leq E(B W A) \leq U B_{6}-k \sigma(B W A) \\
& l b+k \sigma(x) \leq d_{i} \leq u b-k \sigma(x) \quad \text { for } i=1,2, \ldots, 5
\end{array}
$$




$$
\begin{aligned}
& \mu_{z}^{*}=\underset{\mu_{z}}{\arg \max }\left(w^{*} E(G W)+(1-w) * \sigma(G W)\right) \\
& \text { s.t. } \quad Z_{l} \leq \mu_{z_{i}} \leq Z_{u} \quad \text { for } i=1
\end{aligned}
$$

where the bounds $Z_{l}$ and $Z_{u}$ for the mean of the non-design epistemic variable SepQ are calculated by Eq. (10) as given in Section 2.1.1. Note that in Eq. (14), we do not use the robust design constraints, since the objective function in this case is a function of all nondesign epistemic variables.

As mentioned earlier in Section 2, w $\geq 0$ is the weight parameter that represents the relative importance of the objectives and $k$ is a constant that adjusts the robustness of the method against the level of conservatism of the solution. In this paper, $k$ is assumed to be unity.

Variances of the random variables $\boldsymbol{x}$ and $\mathbf{z}$ are estimated as single point values. Confidence intervals for the variances are estimated for each random variable described by the sparse point data. The weight parameter $w$ is varied (from 0 to 1 ) and the optimization problem in Eqs. (13)-(14) are solved iteratively until convergence by the Matlab solver 'fmincon' for different sample sizes $(n)$ of the sparse point data. In each case, the optimization problems converged in less than 5 iterations. Here, 'fmincon' uses a sequential quadratic programming (SQP) algorithm. The estimate of the Hessian of the Lagrangian is updated using the BFGS formula at each iteration. The convergence properties of SQP have been discussed by many authors including Fletcher (1987) and Panier and Tits (1993). 
The solutions are obtained by solving the problem using the upper confidence bound for the variances of the random variables $\boldsymbol{x}$ and $\boldsymbol{z}$. The solutions are presented in Figure 2 .

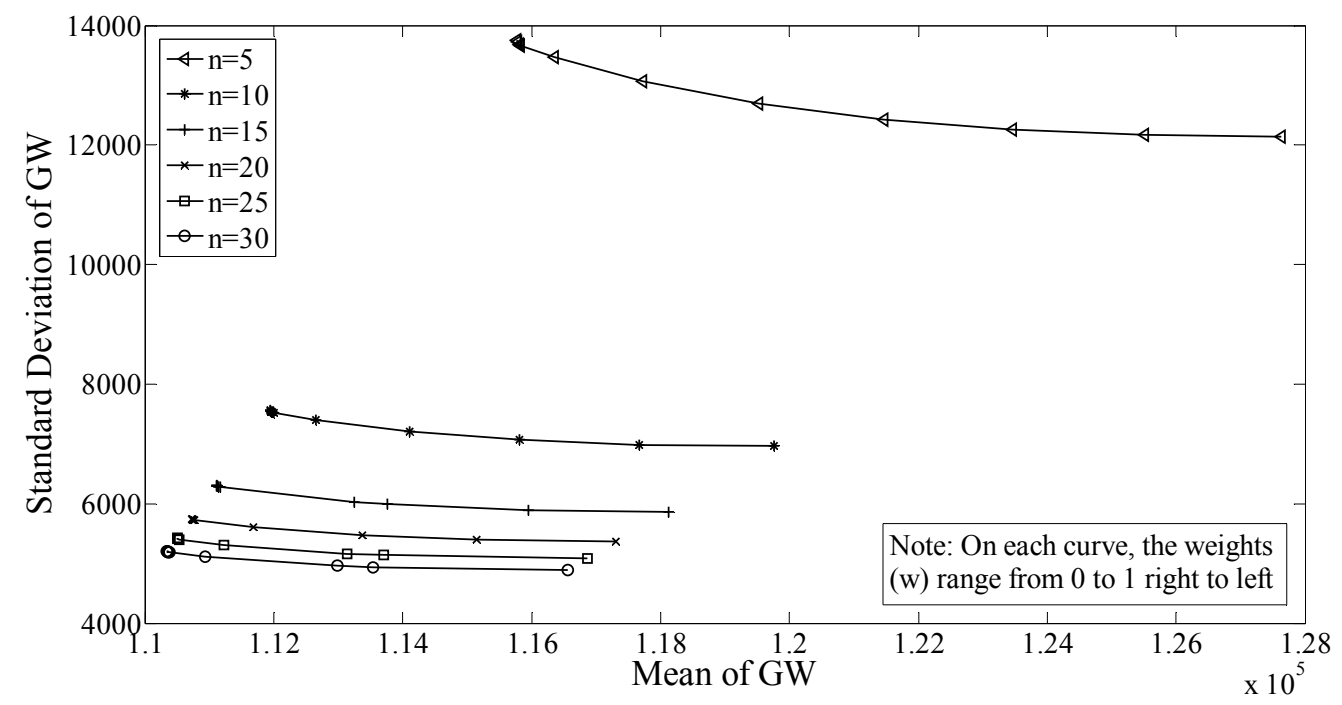

Figure 2: Robustness-based design optimization with sparse data for different sample

$$
\text { sizes }(n)
$$

It is seen in Figure 2 that the solutions become more conservative (i.e., the mean and standard deviation of GW assume higher values) as we add uncertainty to the design problem. It is also seen from Figure 2 that as the sample size $(n)$ increases, both the standard deviation and mean of $\mathrm{GW}$ decrease. As gathering more data reduces data uncertainty, the solutions become less sensitive (i.e., the standard deviation of GW assumes lower value) to the variations of the input random variables as the sample size (n) increases. Also, looking at the mean of $\mathrm{GW}$, it is seen that as the uncertainty decreases with sample size, the optimum mean weight required is less.

\section{2 Determination of optimal sample size for sparse point data}


The optimal sample size formulations are illustrated here for the TSTO design problem. The formulations are relaxed by assuming that standard deviations of the data do not change significantly as sample size changes. To make the problem simpler, we first relax the integer requirement on the optimal sample size $n$ and then round off the solution for $n$ to the nearest integer value. The input variable SepQ is assumed to be a non-design epistemic variable and all the remaining variables are assumed to be design variables. The design bounds for the respective design variables and the analysis outputs remain the same as in Tables 3 and 4.

Therefore, the design problem becomes as follows:

$$
\begin{aligned}
{\left[d^{*}, n_{d}^{*}\right]=} & \arg \min _{d, n_{d}}\left(w^{*} E(G W)+v^{*} \sigma(G W)+(1-w-v) *\left(5 n_{d 1}+10 n_{d 2}+5 n_{d 3}+5 n_{d 4}+4 n_{d 5}+6 n_{e}^{*}\right)\right) \\
\text { s.t. } \quad & L B_{1}+\sigma(G W) \leq E(G W) \leq U B_{1}-\sigma(G W) \\
& L B_{2}+\sigma(E W) \leq E(E W) \leq U B_{2}-\sigma(E W) \\
& L B_{3}+\sigma(P F R) \leq E(P F R) \leq U B_{3}-\sigma(P F R) \\
& L B_{4}+\sigma(V L) \leq E(V L) \leq U B_{4}-\sigma(V L) \\
& L B_{5}+\sigma(V V) \leq E(V V) \leq U B_{5}-\sigma(V V) \\
& L B_{6}+\sigma(B W A) \leq E(B W A) \leq U B_{6}-\sigma(B W A) \\
& l b+k \sigma(\underline{x}) \leq d_{i} \leq u b-k \sigma(\underline{x}) \quad \text { for all } i=1,2, \ldots, 5 \\
& 5 n_{d 1}+10 n_{d 2}+5 n_{3}+5 n_{d 3}+4 n_{d 5}+6 n_{e}^{*} \leq 1050 \\
& n_{d_{j}} \leq 30 \quad \text { for } \mathrm{j}=1,2, \ldots, 5 \\
{\left[\mu_{z}^{*}, n_{e}^{*}\right]=} & \arg \max _{\mu_{z}, n_{e}}\left(w^{*} E(G W)+v^{*} \sigma(G W)+(1-w-v) *\left(5 n_{d_{1}}^{*}+10 n_{d_{2}}^{*}+5 n_{d_{3}}^{*}+5 n_{d_{3}}^{*}+4 n_{d 5}^{*}+6 n^{e}\right)\right) \\
& Z_{l}\left(n_{e}\right) \leq \mu_{z} \leq Z_{u}\left(n_{e}\right) \\
& 5 n_{d_{1}}^{*}+10 n_{d 2}^{*}+5 n_{d_{3}}^{*}+5 n_{d 3}^{*}+4 n_{d 5}^{*}+6 n^{e} \leq 1050 \\
& n_{e_{j}} \leq 30 \quad \text { for } \mathrm{j}=1
\end{aligned}
$$


We have solved this problem for different combinations of weights $w$ and $v$ and the optimal solutions are presented in Table 6. In each case, the optimization problems converged in less than 4 iterations.

Table 6: Objective function values at optimal solutions and optimal sample sizes

\begin{tabular}{|c|c|c|l|l|l|l|l|l|c|c|c|}
\hline \multicolumn{3}{|c|}{ Weights } & \multicolumn{4}{c|}{ Objective function Value } & \multicolumn{5}{c|}{ Optimal Sample Sizes } \\
\hline$w$ & $v$ & $\begin{array}{c}1- \\
w- \\
v\end{array}$ & Mean GW & Std GW & $\begin{array}{l}\text { Total } \\
\text { Cost }\end{array}$ & $n_{d 1}$ & $n_{d 2}$ & $n_{d 3}$ & $n_{d 4}$ & $n_{d 5}$ & $n_{e}$ \\
\hline 0 & 0 & 1 & $1.6118 \mathrm{e}+005$ & $6.3732 \mathrm{e}+004$ & 455.3008 & 5 & 10 & 15 & 8 & 9 & 30 \\
\hline 0.6 & 0.2 & 0.2 & $1.4684 \mathrm{e}+005$ & $5.3219 \mathrm{e}+004$ & 539.8948 & 6 & 10 & 30 & 8 & 10 & 30 \\
\hline 0.5 & 0.4 & 0.1 & $1.4878 \mathrm{e}+005$ & $5.0526 \mathrm{e}+004$ & 593.6961 & 7 & 10 & 30 & 14 & 15 & 30 \\
\hline 0.5 & 0.5 & 0 & $1.5143 \mathrm{e}+005$ & $4.7604 \mathrm{e}+004$ & 886.9363 & 25 & 25 & 30 & 30 & 30 & 15 \\
\hline
\end{tabular}

It is seen in Table 6 that the total cost incurred in obtaining samples is the minimum when we solve the problem giving the maximum importance on the total cost. In this case, we get the most conservative robust design i.e., the mean and the standard deviation of GW assume the maximum of all possible values. Note that the optimal sample size required is also the minimum in this case. As we give more importance on the mean and standard deviation of GW, the total cost and also the optimal sample size increase with a decrease in both the mean and standard deviation of GW.

\section{3 Robustness-based design optimization with sparse point and interval data}

The methodology proposed in Section 2.1 is illustrated here for the same TSTO problem. Here, it is assumed that the design variable ExpRatio is described by sparse point data as given in Table 5, the design variable Payload is described by multiple interval data as given in Table 7 and the design variables SepMach and SepQ are described by single interval data as given in Table 8 . The non-design epistemic variables 
SepAngle and Fineness are described by the sparse point data (as given in Table 5) and the single interval data (as given in Table 7), respectively. The design bounds for the respective design variables and the analysis outputs remain the same as in Tables 3 and 4 .

Table 7: Multiple Interval Data for the random input variables

\begin{tabular}{|l|l|}
\hline Payload & $\begin{array}{l}{[25000,28000],[26000,29000],[25000,29000],[26000,30000],} \\
{[25000,30000]}\end{array}$
\end{tabular}

Table 8: Single Interval Data for the random input variables

\begin{tabular}{|l|l|}
\hline SepMach & {$[9,10]$} \\
\hline SepQ & {$[100,120]$} \\
\hline
\end{tabular}

The design problem is now formulated as follows:

$$
\begin{array}{ll}
d^{*}=\underset{d}{\arg \min }\left(w^{*} E(G W)+(1-w)^{*} \sigma(G W)\right) \\
\text { s.t. } \quad L B_{1}+k \sigma(G W) \leq E(G W) \leq U B_{1}-k \sigma(G W) \\
& L B_{2}+k \sigma(E W) \leq E(E W) \leq U B_{2}-k \sigma(E W) \\
& L B_{3}+k \sigma(P F R) \leq E(P F R) \leq U B_{3}-k \sigma(P F R) \\
& L B_{4}+k \sigma(V L) \leq E(V L) \leq U B_{4}-k \sigma(V L) \\
& L B_{5}+k \sigma(V V) \leq E(V V) \leq U B_{5}-k \sigma(V V) \\
& L B_{6}+k \sigma(B W A) \leq E(B W A) \leq U B_{6}-k \sigma(B W A) \\
& l b+k \sigma(x) \leq d_{i} \leq u b-k \sigma(x) \quad \text { for } i=1,2,3,4 \\
& \quad Z_{l} \leq \mu_{z_{i}} \leq Z_{u} \quad \text { for } i=1,2
\end{array}
$$

where the bounds $Z_{l}$ and $Z_{u}$ for the mean value of the non-design epistemic variable SepAngle are calculated by Eq. (10) as given in Section 2.1.1 and those for the epistemic variable Fineness are calculated by the method described in Section 2.1.3. Note that in 
Eq. (16), we do not use the robust design constraints, since the objective function in this case is a function of all non-design epistemic variables.

Variances of the random variables ExpRatio and SepAngle are estimated as single point values. Confidence intervals for the variances are estimated for each random variable described by sparse point data. Bounds on the variances of the random variables SepMach, SepQ, Fineness, and Payload are estimated by the methods described in Sections 2.1.3. The free parameter $w$ is varied (from 0 to 1) and the optimization problems in Eqs. (15) and (16) are solved iteratively until convergence. In each case, the optimization problems converged in less than 5 iterations. The solutions are obtained by solving the problems using the upper confidence bound on sample variance for the random variables ExpRatio and SepAngle, and the upper bound on sample variances for the random variables Payload, SepMach, SepQ and Fineness. The solutions are presented in Figure 3.

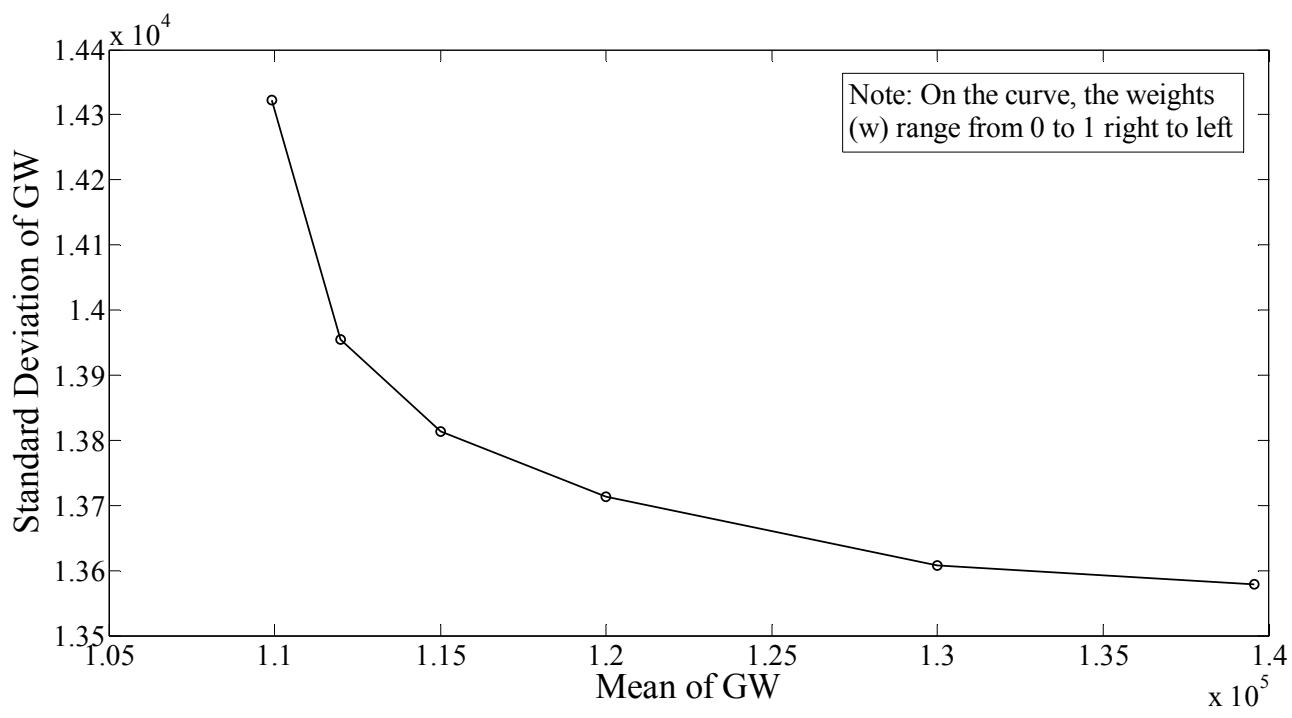

Figure 3: Robustness-based design optimization with non-design epistemic variables 
Figure 3 shows the solutions of the conservative robust design in presence of uncontrollable epistemic uncertainty described through mixed data i.e., both sparse point data and interval data, which is seen frequently in many engineering applications.

\section{Summary and Conclusion}

This paper proposed several formulations for robustness-based design optimization under data uncertainty. Two types of data uncertainty - sparse point data and interval data - are considered. The proposed formulations are illustrated for the upper stage design problem of a TSTO space vehicle. A decoupled approach is proposed in this paper to un-nest the robustness-based design from the analysis of non-design epistemic variables to achieve computational efficiency. As gathering more data reduces uncertainty but increases cost, the effect of sample size on the optimality and the robustness of the solution is also studied. This is demonstrated by numerical examples, which suggest that as the uncertainty decreases with sample size, the resulting solutions become more robust. We have also proposed a formulation to determine the optimal sample size for sparse point data that leads to the solution of the design problem that is least sensitive (i.e., robust) to the variations of design variables. In this paper, we have used the weighted sum approach for the aggregation of multiple objectives and to examine the trade-offs among multiple objectives. Other multi-objective optimization techniques can also be explored within the proposed formulations.

The major advantage of the proposed methodology is that unlike existing methods, it does not use separate representations for aleatory and epistemic uncertainties and does not require nested analysis. Both types of uncertainty are treated in a unified 
manner using a probabilistic format, thus reducing the computational effort and simplifying the optimization problem. The results regarding robustness of the design versus data size are valuable to the decision maker. The design optimization procedure also optimizes the sample size, thus facilitating resource allocation for data collection efforts. Due to the use of a probabilistic format to represent all the uncertain variables, the proposed robustness-based design optimization methodology facilitates the implementation of multidisciplinary robustness-based design optimization, which is a challenging problem in presence of epistemic uncertainty.

\section{Acknowledgement}

This study was supported by funds from NASA Langley Research Center under Cooperative Agreement No. NNX08AF56A1 (Technical Monitor: Mr. Lawrence Green). The support is gratefully acknowledged.

\section{References:}

[1] Agarwal, H, Mozumder, C K, Renaud, J E, and Watson, L T, An inverse-measurebased unilevel architecture for reliability-based design optimization, Struct Multidisc Optim (2007) 33: 217-227.

[2] Bichon, B J, McFarland, J M and Mahadevan, S, Using Bayesian Inference and Efficient Global Reliability Analysis to Explore Distribution Uncertainty, $49^{\text {th }}$ AIAA/ASME/ASCE/AHS/ASC Structures, Structural Dynamics, and Materials Conference, 7 - 10 April 2008, Schaumburg, IL.

[3] Bras, B. A., and Mistree, F., 1993, Robust Design using Compromise Decision Support Problems, Engineering Optimization, Vol. 21, pp. 213-239.

[4] Bras, B. A. and Mistree, F., 1995, A Compromise Decision Support Problem for Robust and Axiomatic Design, ASME Journal of Mechanical Design, Vol. 117, No. 1, pp. 10-19.

[5] Bonett, D G., Approximate confidence interval for standard deviation of nonnormal distributions, Computational Statistics \& Data Analysis 50 (2006) 775 - 782. 
[6] Cagan, J., Williams, B. C., 1993, First-Order Necessary Conditions for Robust Optimality, in ASME Advances in Design Automation, Albuquerque, NM, ASME DEVol. 65-1.

[7] Chen, W., Allen, J. K., Mistree, F. and Tsui, K.-L., 1996, A Procedure for Robust Design: Minimizing Variations Caused by Noise Factors and Control Factors, ASME Journal of Mechanical Design, Vol. 118, pp.478-485.

[8] Chen W, Wiecek MM, Zhang J., Quality utility-a compromise programming approach to robust design, Journal of Mechanical Design (ASME) 1999; 121:179-187.

[9] Chen, W., Sahai, A., Messac, A., Sundararaj, G.J., Exploration of the effectiveness of physical programming in robust design, J. Mech. Des. 122, 155-163, 2000.

[10] Cheng, $\mathrm{H}$ and Sandu, A, Efficient uncertainty quantification with the polynomial chaos method for stiff systems, Mathematics and Computers in Simulation 79 (2009) 3278-3295.

[11] Chiralaksanakul, A., and Mahadevan, S., First-Order Approximation Methods in Reliability-Based Design Optimization, J. Mech. Des.,Volume 127, Issue 5, 2005.

[12] Cojbasic, V., Tomovic, A., Nonparametric confidence intervals for population variance of one sample and the difference of variances of two samples, Computational Statistics \& Data Analysis 51 (2007) 5562 - 5578.

[13] Dai, Z, and Mourelatos, Z P, Incorporating Epistemic Uncertainty in Robust Design, Proceedings of DETC, 2003 ASME Design Engineering Technical Conferences September 2-6, 2003, Chicago, Illinois, USA.

[14] Doltsinis, I, and Kang, Z, Robust design of structures using optimization methods, Comput, Methods Appl. Mech. Engrg. 193 (2004) 2221-2237.

[15] Du, X., and Chen, W., Towards a better understanding of Modeling Feasibility Robustness in Engineering, ASME J. Meach. Des., 2000, 122(4).

[16] Du. X, Sudjianto, A., Chen. W., An Integrated Framework for Optimization Under Uncertainty Using Inverse Reliability Strategy, ASME, 2004.

[17] Du, X, and Beiqing Huang, B, Reliability-based design optimization with equality constraints, Int. J. Numer. Meth. Engng 2007; 72:1314-1331.

[18] Fletcher, R, Practical Methods of Optimization, 2nd ed. John Wiley, New York, 1987. 
[19] Ghanem, R., and Spanos, P., Stochastic Finite Elements: A Spectral Approach, Springer-Verlag, New York, 1991.

[20] Haldar A., Mahadevan S., Probability, Reliability and Statistical Methods in Engineering Design, John Wiley \& Sons, Inc., 2000.

[21] Hong, HP, An efficient point estimate method for probabilistic analysis, Reliability Engineering and System Safety 59 (1998) 261-267.

[22] Huang, B., and Du, X, Analytical robustness assessment for robust design, Struct Multidisc Optim (2007) 34:123-137.

[23] Johnson, N J., Modified $t$ Tests and Confidence Intervals for Assymmetrical Populations, Journal of the American Statistical Association, Vol. 73, No. 363, Sep., 1978, pp. 536-544.

[24] Lee, K-H, and Park, G-J, Robust optimization considering tolerances of design variables, Computers and Structures 79 (2001) 77-86.

[25] Lee. I., Choi. K. K., Du. L., Gorsich. D., Dimension reduction method for reliabilitybased robust design optimization, Computers and Structures, 2008.

[26] Marler, R T, and Arora, J S, Survey of multi-objective optimization methods for engineering, Struct Multidisc Optim 26, 369-395 (2004).

[27] Mavrotas, G, Effective implementation of the e-constraint method in Multi Objective Mathematical Programming problems, Applied Mathematics and Computation 213 (2009) 455-465.

[28] Messac, A., Physical Programming Effective Optimization for Computational Design, AIAA Journal, Vol. 34, No. 1, 1996, pp. 149-158.

[29] Messac, A., Melachrinoudis, E., and Sukam, C. P., Mathematical and Pragmatic Perspectives of Physical Programming, AIAA Journal, Vol. 39, No. 5, 2001, pp. 885893.

[30] Messac, A, and Ismail-Yahaya, A, Multiobjective robust design using physical programming, Struct Multidisc Optim 23, 357-371, 2002.

[31] Oberkampf WL, Helton JC, Joslyn CA, Wojtkiewicz SF, Ferson S, Challenge Problems: uncertainty in system response given uncertain parameters, Reliability Engineering and System Safety, 85 (2004) 11-19.

[32] Panier, E R, and Tits, A L, On combining feasibility, descent and superlinear convergence in inequality constrained optimization, Mathematical Programming, 59:261-276, 1993. 
[33] Park, G-J, Lee, T-H, Lee, KH, and Hwang, K-H, Robust Design: An Overview, AIAA JOURNAL, Vol. 44, No. 1, January 2006.

[34] Parkinson, A., Sorensen, C., and Pourhassan, N., 1993, A General Approach for Robust Optimal Design, Transactions of the ASME, Vol. 115, pp.74-80.

[35] Ramu, P., Qu, X., Youn, B.D., Haftka, R.T. and Choi, K.K. (2006) Inverse reliability measures and reliability-based design optimisation, Int. J. Reliability and Safety, Vol. 1, Nos. 1/2, pp.187-205.

[36] Robert, C. P. and Casella, G., Monte Carlo Statistical Methods. 2nd ed. SpringerVerlag, New York, 2004.

[37] Rosenblueth E (1975), Point estimates for probability moment, Proc Natl Acad Sci U S A 72(10):3812-3814.

[38] Sim M., Robust Optimization, PhD dissertation submitted to the Sloan School of Management, Massachusetts Institute of Technology, June 2004.

[39] Stevenson M D, Hartong A R, Zweber J V, Bhungalia A A, Grandhi R V, Collaborative Design Environment for Space Launch Vehicle Design and Optimization, Paper presented at the RTO AVT Symposium on "Reduction of Military Vehicle Acquisition Time and Cost through Advanced Modeling and Virtual Simulation", held in Paris, France, April 2002.

[40] Sundaresan, S., Ishii, K., and Houser, D. R., A Robust Optimization Procedure with Variations on Design Variables and Constraints, Engineering Optimization, Vol. 24, No. 2, 1995, pp. 101-117.

[41] Taguchi, G., 1993, Taguchi on Robust Technology Development: Bringing Quality Engineering Upstream, ASME Press, New York.

[42] Wei D L, Cui, Z S, and Chen, J, Robust optimization based on a polynomial expansion of chaos constructed with integration point rules, J. Mechanical Engineering Science, Vol. 223 Part C, 2009.

[43] Youn, B D, Choi, K K, and Du, L, Integration of Possibility-Based Optimization and Robust Design for Epistemic Uncertainty, ASME Journal of Mechanical Design, Vol. 129, AUGUST 2007.

[44] Yu, J-C and Ishii, K., 1998, Design for Robustness Based on Manufacturing Variation Patterns, Transactions of the ASME, Vol.120, pp. 196-202.

[45] Zaman, K, Rangavajhala, S, McDonald, PM., and Mahadevan, S, 2009, A probabilistic approach for representation of interval uncertainty, Reliability Engineering and System Safety. (under review) 
[46] Zeleny, M. 1973, Compromise programming, in: Multiple Criteria Decision Making, eds: J. L. Cochrane and M. Zeleny, University of South Carolina Press, Columbia, SC, pp. 262-301.

[47] Zhang, W H, A compromise programming method using multibounds formulation and dual approach for multicriteria structural optimization, Int. J. Numer. Meth. Engng 2003; 58:661-678.

[48] Zhao, Y-G, Ono, T (2000), New point estimates for probability moments,, J Eng Mech 126(4):433-436.

[49] Zhao Y-G, Ang AH-S (2003), System reliability assessment by method of Moments, J Struct Eng 129(10):1341-1349.

[50] Zou, T, and Mahadevan, S, Versatile Formulation for Multiobjective ReliabilityBased Design Optimization, J. Mech. Des. 128, 1217 (2006). 